\title{
Separating quasi-convex subgroups in 7-systolic groups
}

\author{
Frédéric Haglund and Jacek Świątkowski*
}

\begin{abstract}
Let $\Gamma$ be a group acting without inversions and simply transitively on the topdimensional simplices of some simply-connected simplicial complex $X$ with "simplicial negative curvature". Then the quasi-convex subgroups of $\Gamma$ are convex-cocompact. Furthermore, if the action of $\Gamma$ on $X$ satisfies some additional condition called "extra-tilability", the quasiconvex subgroups of $\Gamma$ are separable, i.e. every such subgroup is the intersection of finite index subgroups. The latter result applies to a large class of "simplicially negatively curved" groups recently constructed by Januszkiewicz and the second author.
\end{abstract}

Mathematics Subject Classification (2000). 20F67, 20E07, 20 F65.

Keywords. Separable subgroup, quasi-convex subgroup, word hyperbolic group, systolic group.

\section{Introduction}

Separability properties attracted attention since the emergence of combinatorial group theory. The simplest such property is residual finiteness. A group is residually finite if the intersection of all finite index subgroups is the trivial subgroup $\{1\}$. More generally a subgroup $H<G$ is separable if it can be expressed as the intersection of a certain family of finite index subgroups of $G$. The residual finiteness of $G$ is equivalent to the separability of the trivial subgroup $\{1\}<G$.

When a group $G$ is residually finite, then the word problem in $G$ is solvable, and furthermore $G$ is hopfian (that is: every surjective homomorphism of $G$ to itself is an automorphism). We refer the reader to [14] for a survey of those and other early developments concerning residual finiteness. Separability of a subgroup $H<$ $G$ implies that the membership problem for $H$ is decidable [4]. The geometric interpretation of separability in case of $\pi_{1}$-injective immersions of surfaces in 3manifolds (with separable image) is that it is possible to remove self-intersections by passing to finite sheeted covers, see [17].

\footnotetext{
* Research supported by grants from NSERC and FCAR and the MNiSW grants 2 P03A 01725 and N201 012 32/0718.
} 
Separability properties are difficult to establish or disprove. For example, it is not known whether there exists a word-hyperbolic group which is not residually finite. The property that every finitely generated subgroup is separable (so called subgroup separability) is established for free groups [7], surface groups [17], finitely generated nilpotent groups [15] and some other classes of groups (see [5], [6], [16], [19] and the references cited there).

A few years ago D. Wise initiated the study of the property (denoted QCS below) of separability for all quasi-convex subgroups of a group, rather than for all finitely generated subgroups. The quasi-convexity of a subgroup, in general, depends on a choice of a metric space on which the group acts geometrically (e.g. a Cayley graph). However, in case of word-hyperbolic groups (which we will be interested in) the property is intrinsic. We recall this notion in Definitions 5.4 and 5.6.

Hsu and Wise indicated in [11] examples of groups that are QCS and not subgroup separable. Wise showed the importance of the question about QCS for the problem of residual finiteness of word-hyperbolic groups ([13], [Wi3], 11.8). The question about QCS seems to be well posed for CAT(0) groups, since their quasi-convex subgroups are known to have decidable membership problem (and the same is true for separable subgroups). QCS is established for graph products of finite groups [9], for certain class of Artin groups called tree groups [11], for Bianchi groups [1], and for certain vast class of groups that includes acute angled $n$-gons of finite groups with $n \geq 4$ [20]. On the other hand, QCS does not hold for all CAT(0) groups since there are compact CAT(0) squared 2-complexes whose fundamental groups are not even residually finite (see [18], [3]). In [10] it is proved that the fundamental group of a negatively curved cube complex is QCS if and only if it has a virtual embedding as a "convex cocompact" subgroup into some right-angled Coxeter group.

In this paper we study property QCS for systolic groups. These groups were introduced recently in [12] as groups acting in a geometric way on simply connected simplicial complexes that are simplicially nonpositively curved. Simplicial nonpositive curvature is a purely combinatorial local condition (introduced in [8] and [12]) that mimics classical nonpositive curvature, and has similar topological and group theoretic consequences. In fact, there is an integer parameter $k \geq 6$ that defines a sequence of conditions called $k$-systolicity. Simplicial nonpositive curvature corresponds to 6-systolicity (abbreviated to systolicity), and every $m$-systolic complex or group is $k$-systolic when $m \geq k$. It turns out that 7-systolic groups are wordhyperbolic. For every $k \geq 6$ there are examples of $k$-systolic groups of arbitrarily large cohomological dimension. Examples of such groups, constructed in [12], are the fundamental groups (or direct limits) of simplices of finite groups satisfying certain conditions that we call local $k$-largeness (related to $k$-systolicity) and local extra-tilability. Our inductive constructions of these groups involve their residual finiteness, so in particular the following holds. 
Theorem (Proposition 19.1 in [12]). Let $\mathcal{E}$ be a locally 6-large and locally extratilable simplex of finite groups. Then $\mathcal{E}$ is developable and the direct limit of $\mathcal{E}$ is a residually finite group.

The main result of the present paper, Theorem A below, considerably strengthens the residual finiteness statement in the above theorem.

Theorem A (Theorem 6.1). Let $\mathcal{E}$ be a locally 7-large and locally extra-tilable simplex of finite groups. Then its direct limit has separable quasi-convex subgroups.

Note that simplices of groups satisfying assumptions of Theorem A exist in arbitrary dimension, and that the resulting groups satisfying QCS occur in arbitrary cohomological dimension.

Our proof of Theorem A follows the geometric method of Scott [17]. It is based on a result of independent interest, Theorem B below, concerning convex cocompactness of quasi-convex subgroups in arbitrary 7-systolic groups. In this result we use the notion of a convex subcomplex in a systolic complex, two different aspect of which were introduced and studied in [8] and [12] respectively. (The two notions of convexity are in fact equivalent, see Proposition D below.) More precisely, we prove the following.

Theorem B (Corollary 5.8). Let G be a 7-systolic group, i.e., a group acting properly discontinuously and cocompactly, by simplicial automorphisms, on certain 7-systolic simplicial complex $X$. Let $H<G$ by any quasi-convex subgroup. Then there is a convex subcomplex $Y \subset X$ which is invariant and cocompact under the action of $H$.

Apart from application to proving Theorem A, Theorem B (together with the fact that a convex subcomplex of a $k$-systolic complex is $k$-systolic, see Lemma 2.6) implies the following.

Corollary C. For any $k \geq 7$, a quasi-convex subgroup of a $k$-systolic group is $k$-systolic.

This corollary is a special case of the following result announced by D. Wise [21]: for any $k \geq 6$, a finitely presented subgroup of a $k$-systolic group is $k$-systolic. Wise's proof is different and uses the tower argument.

In Sections 4 and 5 we prove certain results concerning systolic geometry. These results are useful in proving our main results: Theorems A and B. However, they also have generally useful nature and complement the set of basic geometric properties of systolic simplicial complexes.

Two main results of Section 4 concern geodesics. For us a geodesic in a simplicial complex $X$ between some two vertices is a polygonal curve in the 1-skeleton of $X$ that connects these vertices and has the smallest possible number of edges. A subcomplex 
$Y \subset X$ is geodesically convex if it is full and any geodesic in $X$ between any two vertices of $Y$ is contained in $Y$ (this is the notion of convexity considered in [8]). In systolic complexes $X$ this "global" convexity property (i.e. geodesical convexity as above) turns out to coincide with the "local" property of convexity (as considered in [12] and recalled in Section 2, see Definition 2.5). Namely, we prove the following.

Proposition D (Proposition 4.9). A subcomplex $Y$ in a systolic simplicial complex $X$ is geodesically convex iff it is convex.

The next result (see Lemma 3.4 in [8]) concerns geodesics in 7-systolic complexes. The 2-dimensional case seems to be well known in small cancellation theory.

Proposition E (Proposition 4.7). Let $\left(v_{0}, v_{1}, \ldots, v_{n-1}, v_{n}\right)$ and $\left(v_{0}, v_{1}^{\prime}, \ldots, v_{n-1}^{\prime}, v_{n}\right)$ be two geodesics in a 7 -systolic simplicial complex $X$ between vertices $v_{0}, v_{n}$. Then for each $1 \leq i \leq n-1$ the vertices $v_{i}$ and $v_{i}^{\prime}$ either coincide or are connected with an edge of $X$.

The main geometric result of this paper is proved in Section 5 and concerns convex neighbourhoods of quasi-convex subsets in 7-systolic complexes. For any subcomplex $Y$ of a simplicial complex $X$, let $B(Y, X)$ denote the union of all simplices of $X$ meeting $Y$. For any integer $n \geq 1$ define inductively the neighbourhoods $B_{n}(Y, X)$ by $B_{1}(Y, X)=B(Y, X)$ and $B_{n+1}(Y, X)=B\left(B_{n}(Y, X), X\right)$.

Theorem $\mathbf{F}$ (Theorem 5.5). Let $Y$ be a quasi-convex subcomplex of a 7-systolic complex $X$. Then there is a natural number $N$ such that for any $n \geq N$ the neighbourhood $B_{n}(Y, X)$ is a convex subcomplex of $X$.

Theorem F answers the last question in [8] and immediately implies Theorem B.

\section{Systolic complexes: definitions and basic results}

In this section we recall definitions and some results concerning systolic complexes and groups. All simplicial complexes $X$ in this paper are assumed to be locally finite dimensional in the following sense: for every vertex $v$ of $X$ there is a bound on the dimension of simplices in $X$ that contain $v$. Clearly, this class contains all finite dimensional and all locally finite simplicial complexes, which are most interesting for us.

2.1. Local $\boldsymbol{k}$-largeness, $\boldsymbol{k}$-systolicity and consequences. Systolicity is a purely combinatorial condition for simplicial complexes (and their automorphism groups) that resembles nonpositive curvature. Main references for this subject are [8] and [12]. 
A simplicial complex $X$ is flag if every finite set of its vertices pairwise connected by edges spans a simplex of $X$. A cycle in a simplicial complex is a subcomplex homeomorphic to the circle $S^{1}$. The length $|\gamma|$ of a cycle $\gamma$ is the number of its edges. We say that a cycle in $X$ has a diagonal if some two nonconsecutive vertices in this cycle are connected by an edge in $X$.

Let $k \geq 5$ be a natural number. A simplicial complex $X$ is $k$-large if it is flag and every cycle in $X$ of length $4 \leq|\gamma|<k$ has a diagonal. A simplicial complex is locally $k$-large if its links at all (nonempty) simplices are $k$-large. A simplicial complex is $k$-systolic if it is locally $k$-large, connected and simply connected. Since the case of $k=6$ is particularly important, we abbreviate the term "6-systolic" to an easier pronounced term systolic.

We view $k$-largeness as local curvature condition for links of a complex, and local $k$-largeness as a kind of curvature bound from above. We sometimes call local 6-largeness simplicial nonpositive curvature since it yields similar consequences as metric nonpositive curvature. A systolic complex is then the simplicial analogue of what is called a CAT(0) or Hadamard space.

A straightforward consequence of the above definitions is the following.

Lemma 2.1. Let $X$ be a simplicial complex.

(1) If $k \geq m$ and $X$ is $k$-large then $X$ is also $m$-large.

(2) If $k \geq m$ and $X$ is $k$-systolic then $X$ is also $m$-systolic.

An example of a local-to-global phenomenon related to simplicial nonpositive curvature is the following.

Proposition 2.2 (see Proposition 1.4 in [12]). For $k \geq 6$ every $k$-systolic complex is $k$-large.

The next result exhibits analogy between systolicity and nonpositive (or even negative) curvature.

Theorem 2.3. (1) (See Theorem 4.1 (1) in [12].) If $X$ is a finite dimensional systolic simplicial complex then $X$ is contractible.

(2) (See Theorem 2.1 in [12].) If $X$ is a 7-systolic simplicial complex then the 1-skeleton of $X$, equipped with the geodesic metric for which every edge has length 1 , is $\delta$-hyperbolic with $\delta=\frac{5}{2}$.

Let us mention that there is no obvious relationship between local 6-largeness (simplicial nonpositive curvature) and nonpositive curvature in metric sense. However, $k$-systolicity for $k$ sufficiently large implies metric nonpositive curvature (see Section 14 in [12]). 
A group is $k$-systolic if it acts by simplicial automorphisms, properly discontinuously and cocompactly, on a $k$-systolic simplicial complex. It is systolic, if it is $k$-systolic with $k=6$. As a consequence of Theorem 2.3(2) above we get the following result.

Corollary 2.4. Every 7-systolic group is word-hyperbolic.

As shown in [12] there exist many $k$-systolic groups, for every $k \geq 6$, in arbitrary dimension. For example, they arise as fundamental groups of certain simplices of groups, which is explained also in the next section (Proposition 3.6 and Proposition 3.7).

2.2. Convexity and saliency. We now describe local convexity phenomena in systolic complexes. We follow Sections 3 and 7 of [12]. For a simplicial complex $X$ we denote its link at a face $\sigma$ by $X_{\sigma}$. The next definition of 3-convexity is a reformulation of the definition given in Section 3 of [12].

Definition 2.5. A subcomplex $Q$ in a 6-large simplicial complex $X$ is 3-convex if it is a full subcomplex and for any path $\left(v_{1}, v_{2}, v_{3}\right)$ in the 1-skeleton of $X$, if $v_{1}, v_{3}$ are contained in $Q$ and not connected with an edge in $X$ then $v_{2} \in Q$. A subcomplex $Y$ in a systolic complex $X$ is convex if it is connected and for every vertex $v \in Q$ the link $Q_{v}$ is 3-convex in the corresponding link $X_{v}$.

In Section 4 we will prove that convexity is equivalent to geodesic convexity, as defined in the introduction (see Proposition 4.9).

Among various properties of the above combinatorial notion of convexity one of interest for us is the following (see Lemma 7.2 and Fact 1.2(2) in [12]).

Lemma 2.6. Any convex subcomplex in a systolic complex is full and contractible. In particular, for any $k \geq 6$, any convex subcomplex in a $k$-systolic complex is itself $k$-systolic.

It turns out that another notion, slightly stronger than convexity, is very useful. In [12] this notion is called strong convexity, but here we will use the term salient, as in [8]. Given a subcomplex $Y$ in a simplicial complex $X$, its neighbourhood in $X$, denoted $B(Y, X)$, is the union of all simplices of $X$ that intersect $Y$.

Definition 2.7. A subcomplex $Y$ in a systolic simplicial complex $X$ is salient if it is connected and for every simplex $\sigma$ in $Y$ either we have equality $Y_{\sigma}=X_{\sigma}$ or there is a simplex $\tau$ in $X_{\sigma}$ such that $Y_{\sigma}=B\left(\tau, X_{\sigma}\right)$.

The next two results indicate important relationships between the notions of salient and convex subcomplexes. 
Lemma 2.8 (see Corollary 7.11 in [12]). Every salient subcomplex of a systolic complex $X$ is convex in $X$.

Theorem 2.9 (see Corollary 7.12 in [12]). Let $Y$ be a convex subcomplex in a systolic complex $X$. Then its neighbourhood $B(Y, X)$ is a salient subcomplex of $X$.

We will use the following simplified definition of saliency:

Lemma 2.10. A connected subcomplex $Y$ in a systolic simplicial complex $X$ is salient ifffor every vertex $v \in Y$ either $Y_{v}=X_{v}$ or $Y_{v}=B\left(\tau, X_{v}\right)$ for some simplex $\tau \subset X_{v}$.

Proof. We need to show that, if links of $Y$ at vertices are as above then links at other simplices also have the desired form. Let $\sigma$ be a simplex of $Y$ which is not a vertex, and let $\sigma=v * \rho$. We then have $Y_{\sigma}=\left(Y_{v}\right)_{\rho}$. If $Y_{v}=X_{v}$, we have also $Y_{\sigma}=X_{\sigma}$, so that $Y_{\sigma}$ is as desired. If $Y_{v}=B\left(\tau, X_{v}\right)$, we get $Y_{\sigma}=\left[B\left(\tau, X_{v}\right)\right]_{\rho}$. Since the neighbourhood of $\tau$ in $X_{v}$ has the same links as the corresponding neighbourhood in the universal cover of $X_{v}$, and the latter neighbourhood is salient by Theorem 2.9, we conclude that the link $Y_{\sigma}$ is as desired.

2.3. Balls and spheres. We now turn to describing balls and spheres in systolic complexes. Recall that for any integer $n \geq 1$ and any subcomplex $Y$ of a simplicial complex $X$ we define inductively the neighbourhoods $B_{n}(Y, X)$ by $B_{1}(Y, X)=$ $B(Y, X)$ and $B_{n+1}(Y, X)=B\left(B_{n}(Y, X), X\right)$.

We state without proof the following easy observations concerning neighbourhoods in simplicial complexes.

Lemma 2.11. Let $X$ be a simplicial complex and $Y$ a subcomplex. Denote by $Y^{0}$ the set of vertices of $Y$.

(1) For any $n \geq 1$ we have $B_{n}(Y, X)=\bigcup_{y \in Y^{0}} B_{n}(y, X)$.

(2) Let $x$ be a vertex of $X$ with $d(x, Y)=n$. Denote by $\operatorname{Proj}_{Y}(x)$ the set of those vertices of $Y$ which are at distance $n$ from $x$. Then

$$
\left[B_{n}(Y, X)\right]_{x}=\bigcup_{y \in \operatorname{Proj}_{Y}(x)}\left[B_{n}(y, X)\right]_{x}
$$

Given a vertex $v$ in a simplicial complex $X$, the balls in $X$ centered at $v$ are the neighbourhoods $B_{n}(\{v\}, X)$ (we rather denote them by $B_{n}(v, X)$ and we set $\left.B_{0}(v, X)=\{v\}\right)$. We also define the distance between two vertices of $X$ as the minimal length of a combinatorial path in the 1-skeleton of $X$ joining the two vertices.

The remaining part of this section lists properties of balls and spheres in systolic complexes. 
Lemma 2.12. Let $X$ be a systolic simplicial complex and let $B=B_{n}(v, X)$ be any ball in $X$, for some $n \geq 1$. Then

(1) $B$ is a salient (and hence also convex) subcomplex in $X$.

(2) $B$ is a full subcomplex in $X$ and it is spanned by the set of vertices at distance $\leq n$ from $v$.

Part (1) of the lemma follows from Lemma 2.8 and Theorem 2.9, while part (2) from Lemma 2.6.

Let $X$ denote a simplicial complex and let $v$ be a vertex of $X$. The sphere $S_{n}(v, X)$ is the full subcomplex of $X$ spanned by the set of vertices at distance $n$ from $v$. Since balls in systolic complexes are full, we get the following:

Corollary 2.13. Let $X$ be a systolic simplicial complex and $v$ a vertex of $X$. Then for any natural $n$ we have $S_{n}(v, X) \subset B_{n}(v, X)$.

Given a simplex $\sigma$ in a simplicial complex $X$, the residue of $\sigma$ in $X$ (called also the star of $\sigma$ in $X)$, denoted $\operatorname{Res}(\sigma, X)$, is the subcomplex of $X$ equal to the union of all simplices of $X$ that contain $\sigma$.

Lemma 2.14 (see Corollary 7.9 (1) in [12]). Let $X$ be a systolic simplicial complex and $v$ a vertex of $X$. Then for any natural number $n \geq 1$ and any simplex $\rho \subset S_{n+1}(v, X)$ the intersection $B_{n}(v, X) \cap \operatorname{Res}(\rho, X)$ is nonempty and it is a single simplex of $X$.

We will call the simplex $B_{n}(v, X) \cap \operatorname{Res}(\rho, X)$ as above the projection of $\rho \subset$ $S_{n+1}(v, X)$ onto $S_{n}(v, X)$. Projections are useful to describe links of balls, as in the following.

Lemma 2.15 (see Corollary $7.9(2)$ in [12]). Let $x, y$ be two vertices at distance $n$ in a systolic simplicial complex $X$, and let $\sigma$ be the projection of $x$ on the sphere $S_{n-1}(y, X)$. Then $\left[B_{n}(y, X)\right]_{x}=B\left(\sigma, X_{x}\right)$.

\section{Extra-tilability and local extra-tilability for group actions}

For any group $\Gamma$ acting on a simplicial complex $X$ and for any simplex $\sigma$ of $X$ we denote by $\Gamma_{\sigma}$ the stabilizer of $\sigma$ in $\Gamma$.

Definition 3.1. Let $\Gamma$ denote a group acting by simplicial automorphisms on a simplicial complex $X$. We say that a subcomplex $Y \subset X$ is a strict fundamental domain for the action of $\Gamma$ on $X$ provided the orbit of any simplex of $X$ contains one and only one simplex of $Y$. Furthermore we require that $\Gamma_{\sigma}=\{1\}$ for any simplex $\sigma$ interior to $Y$. 
Note that the second condition is usually not needed (see Definition 12.7 in [2]). But the definition above is more appropriate for our purposes and it simplifies the statements.

The following is clear:

Lemma 3.2. Let $\Gamma$ denote a group acting on a simplicial complex $X$. Assume that the subcomplex $Y \subset X$ is a strict fundamental domain for the action of $\Gamma$ on $X$. Then for any simplex $\sigma$ of $Y$ the subcomplex $Y_{\sigma} \subset X_{\sigma}$ is a strict fundamental domain for the action of $\Gamma_{\sigma}$ on $X_{\sigma}$.

Definition 3.3. (1) Let $X$ be a connected simplicial complex, and let $\Gamma$ be a group acting simplicially on $X$. For any subcomplex $Y \subset X$, we denote by $\Gamma(Y)<\Gamma$ the subgroup generated by the stabilizers $\Gamma_{\sigma}$, with $\sigma$ a maximal simplex of $\partial Y$. We say that $Y$ is a tile for $(\Gamma, X)$ if $Y$ is a strict fundamental domain for $\Gamma(Y)$.

(2) We say that the action of $\Gamma$ on $X$ is chamber transitive if it admits a strict fundamental domain consisting in a single simplex $\Delta$. We say that the action is extra-tilable if for any simplex $\sigma$ of $X$ the neighbourhood $B(\sigma, X)$ is a tile.

(3) We say that the action of $\Gamma$ on $X$ is locally extra-tilable if for any simplex $\sigma$ the action of $\Gamma_{\sigma}$ on $X_{\sigma}$ is extra-tilable.

A result analogous to the following is established in Proposition 18.5 from [12]. For the convenience of the reader we give a detailed argument in this generality.

Theorem 3.4. Let $X$ be a systolic simplicial complex and let $\Gamma$ be a group acting simplicially on $X$. Assume that the action is locally extra-tilable. Then every salient subcomplex is a tile.

To prove this theorem we use the theory of simple complexes of groups, as developed in [2], Chapter II.12.

Proof. Let $Y \subset X$ denote any salient subcomplex. We first define a simple complex of groups $\mathcal{E}^{Y}$ over $Y$ with local groups $\Gamma_{\sigma}^{Y}$. For any maximal simplex $\sigma$ of $\partial Y$ we set $\Gamma_{\sigma}^{Y}=\Gamma_{\sigma}$. For any other simplex $\sigma$ of $\partial Y$ we let $\Gamma_{\sigma}^{Y}$ denote the subgroup of $\Gamma_{\sigma}$ generated by the $\Gamma_{\tau}$ 's, for all maximal simplices $\tau$ of $\partial Y$ containing $\sigma$. For any simplex $\sigma$ in the interior of $Y$ we set $\Gamma_{\sigma}^{Y}=\{1\}$. Clearly when $\sigma_{2} \subset \sigma_{1} \subset Y$ we have $\Gamma_{\sigma_{1}}^{Y} \subset \Gamma_{\sigma_{2}}^{Y}$.

Consider the natural (simple) morphism $m^{Y}: \mathcal{E}^{Y} \rightarrow \Gamma(Y)$ given by the canonical inclusions $\Gamma_{\sigma}^{Y} \subset \Gamma(Y)$, and note that this morphism is injective on local groups (see the end of Definition 12.11 in [2]). Thus the complex of groups $\mathcal{E}^{Y}$ has a development corresponding to the morphism $m^{Y}$. This means that there exists an action of $\Gamma(Y)$ on a (multi)simplicial complex $\bar{X}$ containing $Y$ as a strict fundamental domain, such that the stabilizer of any simplex $\sigma \subset Y \subset \bar{X}$ is precisely $\Gamma_{\sigma}^{Y}$ (see Theorem 12.18 
in [2]). Since by definition the groups $\Gamma_{\sigma}^{Y}$ generate $\Gamma(Y)$, the space $\bar{X}$ is connected ([2], Proposition $12.20(2)$ ). To avoid confusions we denote $\Gamma(Y)$ by $\overline{\Gamma(Y)}$ when it acts on $\bar{X}$.

Since $\Gamma_{\sigma}^{Y} \subset \Gamma_{\sigma}$, there is a natural map sending a point $\bar{\gamma} y \in \bar{X}$ to $\gamma y \in X$, where $\bar{\gamma}=\gamma \in \overline{\Gamma(Y)}=\Gamma(Y)$ and $y \in Y$. In order to get the conclusion it is sufficient to show that this map $\bar{X} \rightarrow X$ is an isomorphism. In fact, since $X$ is simply-connected, it is enough to show that $\bar{X} \rightarrow X$ is a covering. By homogeneity, this reduces to checking that $\bar{X} \rightarrow X$ is a local isomorphism at simplices $\sigma$ of $\partial Y$.

Let $\sigma$ be any simplex of $\partial Y$. By Lemma 3.2, the action of the group $\overline{\Gamma(Y)}{ }_{\sigma}=\Gamma_{\sigma}^{Y}$ on the link $\bar{X}_{\sigma}$ admits $Y_{\sigma}$ as a strict fundamental domain. Furthermore, for any simplex $\tau$ of $Y_{\sigma}$ we see that the stabilizer of $\tau$ in $\overline{\Gamma(Y)}_{\sigma}$ is $\Gamma_{\sigma * \tau}^{Y}$, where $\sigma * \tau$ is the join of $\sigma$ with $\tau$, i.e. the simplex spanned by the union of $\sigma$ and $\tau$. On the other hand, since $Y$ is salient in $X$, there exists a simplex $\rho$ in $Y$ such that $Y_{\sigma}=B\left(\rho, X_{\sigma}\right)$. By assumption the action of $\Gamma$ is locally extra-tilable, so $B\left(\rho, X_{\sigma}\right)$ is a tile for the action of $\Gamma_{\sigma}$ on $X_{\sigma}$. This means that the subgroup $\Gamma_{\sigma}\left(B\left(\rho, X_{\sigma}\right)\right)<\Gamma_{\sigma}$ admits $B\left(\rho, X_{\sigma}\right)=Y_{\sigma}$ as a strict fundamental domain. By definition $\Gamma_{\sigma}\left(B\left(\rho, X_{\sigma}\right)\right)$ is generated by all stabilizers $\left(\Gamma_{\sigma}\right)_{\tau}$, for all maximal simplices $\tau$ of the boundary of $Y_{\sigma}$. But $\left(\Gamma_{\sigma}\right)_{\tau}=\Gamma_{\sigma * \tau}$, and the join $\sigma * \tau$ varies all over the set of maximal simplices of $\partial Y$ containing $\sigma$. Thus in fact $\Gamma_{\sigma}\left(B\left(\rho, X_{\sigma}\right)\right)=\Gamma_{\sigma}^{Y}$.

We observe that the same group $\Gamma_{\sigma}^{Y}$ acts on two simplicial complexes $\left(\bar{X}_{\sigma}\right.$ and $X_{\sigma}$ ) with the same strict fundamental domain (namely $Y_{\sigma}$ ). Thus the natural map $\bar{X}_{\sigma} \rightarrow X_{\sigma}$ is an equivariant isomorphism, provided we prove that stabilizers of simplices in the fundamental domain are the same for the two actions (see for example Proposition 12.20(1) in [2]). Let $\tau$ be a simplex of $Y_{\sigma}$. We have noticed that the stabilizer of $\tau$ in $\overline{\Gamma(Y)}_{\sigma}$ is $\Gamma_{\sigma * \tau}^{Y}$. Moreover, we clearly have $\Gamma_{\sigma * \tau}^{Y} \subset \Gamma_{\sigma * \tau} \cap \Gamma_{\sigma}^{Y}=$ $\left(\Gamma_{\sigma}^{Y}\right)_{\tau}$. It remains to check the reverse inclusion. There is nothing to prove if $\tau$ is in the interior of $Y_{\sigma}$, for in this case $\left(\Gamma_{\sigma}^{Y}\right)_{\tau}=\{1\}$. Assume that $\tau \subset \partial Y_{\sigma}$. By saliency there exists a simplex $\rho^{\prime}$ in the interior of $Y$ such that $Y_{\sigma * \tau}=B\left(\rho^{\prime}, X_{\sigma * \tau}\right)$. As we have seen, $Y_{\sigma * \tau}$ is a strict fundamental domain for the action of $\Gamma_{\sigma * \tau}^{Y}$ on $X_{\sigma * \tau}$. Thus for any $\gamma \in \Gamma_{\sigma}^{Y}$ such that $\gamma \tau=\tau$, there exists a (unique) $\gamma^{\prime} \in \Gamma_{\sigma * \tau}^{Y}$ such that $\gamma^{\prime-1} \gamma \rho^{\prime} \subset Y_{\sigma * \tau}$. Since $\gamma^{-1} \gamma \in \Gamma_{\sigma}^{Y}$ and $Y_{\sigma}$ is a strict fundamental domain for the action of $\Gamma_{\sigma}^{Y}$, we have $\gamma^{\prime-1} \gamma \rho^{\prime}=\rho^{\prime}$ and thus in fact $\gamma=\gamma^{\prime}$, since $\rho^{\prime}$ is interior to $Y_{\sigma}$ too.

An effective source of groups acting in an extra-tilable (and locally extra-tilable) way is a construction of appropriate simplices of groups given in [12]. We recall briefly the outcome of this construction. For the general theory of simple complexes of groups we refer the reader to [2], Chapter II.12. In particular, the notion of a local development is explained there in Construction 12.24, p. 387.

Definition 3.5. Let $\mathcal{E}$ be a simplex of groups, let $\Delta$ denote the underlying simplex, and for any face $\sigma$ of $\Delta$ let $G_{\sigma}$ denote the local group of $\mathcal{E}$ at $\sigma$. A simplex of groups 
is locally $k$-large if all of its local developments are $k$-large simplicial complexes. A simplex of groups is locally extra-tilable if the action of every local group $G_{\sigma}$ on the local development at $\sigma$ is extra-tilable. When the local group $\mathscr{E}_{\Delta}$ is trivial, we say that the simplex of groups is $\partial$-supported.

Proposition 3.6. Given an integer $k \geq 6$, let $\mathcal{E}$ be a $\partial$-supported, locally $k$-large simplex of finite groups, and let $\Gamma$ denote its fundamental group. Then $\mathcal{G}$ is developable, its universal cover is $k$-systolic and the action of $\Gamma$ on the universal cover is chamber-transitive and properly discontinuous. In particular, if $k \geq 7$ then $\Gamma$ is word-hyperbolic. If furthermore $\mathcal{G}$ is locally extra-tilable, then the action of $\Gamma$ on the universal cover is locally extra-tilable.

Proof. The developability of $\mathcal{E}$ follows by Corollary 17.4 in [12]. The fact that any maximal simplex $\Delta$ in the universal cover is then a strict fundamental domain for $\Gamma$ is clear ( $\Delta$ has trivial stabilizer since $\mathcal{E}$ is $\partial$-supported). Proper discontinuity follows from finiteness of local groups. Local $k$-largeness and local extra-tilability follow from the corresponding assumptions for local developments. Consequently, under the local 7-largeness assumption, the universal cover is 7-systolic and $\Gamma$ is then wordhyperbolic by Corollary 2.4. Finally, the last assertion of the statement is clear by comparing the corresponding notions of extra-tilability.

Locally 7-large and locally extra-tilable simplices of finite groups are the main objectives of our study in this paper. The next proposition shows the existence of great many of such simplices of groups.

Proposition 3.7 (Proposition 19.1 in [12]). Let $\Delta$ be a simplex (ofarbitrarydimension) and suppose that for any codimension 1 face $s$ of $\Delta$ we are given a finite group $A_{s}$. Then for any $k \geq 6$ there exists a $\partial$-supported simplex of finite groups $\mathcal{G}$ over $\Delta$, with local groups $G_{\sigma}$, which is locally $k$-large, locally extra-tilable and such that $G_{s}=A_{s}$ for each codimension 1 face $s$ in $\Delta$.

\section{Systolic complexes: further results}

In this section we derive useful properties of geodesics in systolic complexes.

Definition 4.1. A combinatorial path of $X$ is a sequence $\gamma=\left(v_{0}, \ldots, v_{n}\right)$ of vertices of $X$ such that for each $i=0, \ldots, n-1$ the vertices $v_{i}, v_{i+1}$ span an edge of $X$. The path $\gamma$ is said to have length $n$. The endpoints of $\gamma$ are $v_{0}$ and $v_{n}$. Given combinatorial paths $\gamma=\left(v_{0}, \ldots, v_{n}\right)$ and $\gamma^{\prime}=\left(v_{n}, \ldots, v_{n+m}\right)$, we denote by $\gamma \cdot \gamma^{\prime}$ their concatenation $\left(v_{0}, \ldots, v_{n}, v_{n+1}, \ldots, v_{n+m}\right)$.

The combinatorial distance between two vertices $v, w$ is the minimum of the lengths of paths with endpoints $v, w$; we will denote it by $d(v, w)$. A combinatorial 
path with endpoints $v, w$ minimizing the length is called a geodesic of $X$ between $v$ and $w$.

\subsection{Ladders and bigons}

Definition 4.2. A ladder is an ordered pair $\left(\gamma=\left(v_{0}, \ldots, v_{n}\right), \gamma^{\prime}=\left(v_{0}^{\prime}, \ldots, v_{n}^{\prime}\right)\right)$ of (combinatorial) geodesics of $X$ (with the same length) such that:

(1) for each $i=0, \ldots, n-1$ the subset $\left\{v_{i}, v_{i}^{\prime}, v_{i+1}\right\}$ spans a simplex,

(2) for each $i=1, \ldots, n$ the subset $\left\{v_{i-1}^{\prime}, v_{i}^{\prime}, v_{i}\right\}$ spans a simplex.

Note that when $\left(\gamma, \gamma^{\prime}\right)$ is a ladder, in general $\left(\gamma^{\prime}, \gamma\right)$ is not a ladder.

Definition 4.3. A bigon is an ordered pair $\left(\gamma=\left(v_{0}, \ldots, v_{n}\right), \gamma^{\prime}=\left(v_{0}^{\prime}, \ldots, v_{n}^{\prime}\right)\right)$ of (combinatorial) geodesics of $X$ (with the same length) such that $v_{0}=v_{0}^{\prime}$ and $d\left(v_{n}, v_{n}^{\prime}\right) \leq 1$. We say that a bigon is $k$-thin whenever we have $d\left(v_{i}, v_{i}^{\prime}\right) \leq k$ for $i=0, \ldots, n$.

Clearly, a ladder with $v_{0}=v_{0}^{\prime}$ is a 1-thin bigon.

Lemma 4.4. Let $X$ be a systolic complex and let $\gamma=\left(v_{0}, \ldots, v_{n}\right)$ be a geodesic of $X$. Let $w$ denote some vertex of $X$ such that $d\left(v_{0}, w\right)=n$ and $d\left(w, v_{n}\right) \leq 1$. Then there exists a geodesic $\gamma^{\prime}=\left(v_{0}^{\prime}, \ldots, v_{n}^{\prime}\right)$, with $v_{0}^{\prime}=v_{0}$ and $v_{n}^{\prime}=w$, such that $\left(\gamma, \gamma^{\prime}\right)$ is a ladder.

Proof. The lemma is obviously true for $n=0$. We argue by induction on $n \geq 1$. For $n=1$ the flagness of $X$ implies the statement. For $n>1$, if $w=v_{n}$, we may take $\gamma^{\prime}=\gamma$. If $\left\{w, v_{n}\right\}$ spans an edge $e$ in $X$, let $u$ denote a vertex of the projection of $e \subset S_{n}\left(v_{0}\right)$ onto $S_{n-1}\left(v_{0}\right)$ (see Lemma 2.14 and the definition after it). Then $\left\{u, w, v_{n}\right\}$ spans a simplex. Since $v_{n-1}$ and $u$ are in the projection of $v_{n}$ onto $S_{n-1}\left(v_{0}\right)$, we see that $\left\{v_{n-1}, u, v_{n}\right\}$ spans a simplex too. By induction, there is a geodesic $\gamma^{\prime \prime}$ from $v_{0}$ to $u$ such that $\left(\left(v_{0}, v_{1}, \ldots, v_{n-1}\right), \gamma^{\prime \prime}\right)$ is a ladder. It follows that $\left(\gamma, \gamma^{\prime \prime} .(u, w)\right)$ is also a ladder, and the lemma holds for $\gamma^{\prime}=\gamma^{\prime \prime} .(u, w)$.

Corollary 4.5. Let $X$ be a systolic complex and let $\gamma=\left(v_{0}, \ldots, v_{n}\right)$ be a geodesic in $X$, with $n \geq 1$. Let $u$ denote any vertex of $X$ such that $d\left(v_{0}, u\right)=n-1$ and $d\left(u, v_{n}\right)=1$. Then there exists a geodesic $\gamma^{\prime}$ from $v_{0}$ to $v_{n}$, such that $\left(\gamma, \gamma^{\prime}\right)$ is a ladder, and the two last vertices of $\gamma^{\prime}$ are $u$ and $v_{n}$.

Proof. Since $u$ and $v_{n-1}$ belong to the projection of $v_{n} \in S_{n}\left(v_{0}\right)$ onto $S_{n-1}\left(v_{0}\right)$, we know that $\left\{u, v_{n-1}\right\}$ spans a simplex. By Lemma 4.4, there exists a geodesic $\gamma_{1}^{\prime}$ from $v_{0}$ to $u$ such that $\left(\left(v_{0}, v_{1}, \ldots, v_{n-1}\right), \gamma_{1}^{\prime}\right)$ is a ladder. Then $\left(\gamma, \gamma_{1}^{\prime} .\left(u, v_{n}\right)\right)$ is a ladder, and $\gamma_{1}^{\prime} .\left(u, v_{n}\right)$ has the required properties. 
Under assumption of 7-systolicity we will get stronger properties of geodesics. To do this, we need a preparatory lemma.

Lemma 4.6. Let $X$ be a 7-large complex. Let $\sigma, \tau$ denote two simplices of $X$. Assume that $\tau \cap B(\sigma, X)=\emptyset$. Then $B(\sigma, X) \cap B(\tau, X)$ is either empty or a simplex.

Proof. Note that the hypothesis $\tau \cap B(\sigma, X)=\emptyset$ in fact implies $\sigma \cap B(\tau, X)=\emptyset$. Assume that $s, t$ are two distinct vertices of $B(\sigma, X) \cap B(\tau, X)$. Then each of them is disjoint from $\sigma$ and $\tau$, but adjacent to some vertices of $\sigma$, and also to some vertices of $\tau$.

Assume that $s, t$ are adjacent to the same vertex $p$ of $\sigma$. If they are also adjacent to the same vertex $q$ of $\tau$ then $(p, s, q, t)$ is a cycle in $X$ of length 4 . Since $p$ and $q$ are not adjacent by the assumption, 7-largeness of $X$ implies that $s$ and $t$ are adjacent. If $s, t$ are adjacent to distinct vertices $q, r$ of $\tau$ then $(p, s, q, r, t)$ is a cycle in $X$ of length 5 , and $(s, t)$ is the only possible diagonal for this cycle. Again, by 7-largeness of $X, s$ and $t$ are adjacent.

Now consider the case when $s, t$ are adjacent to distinct vertices in both $\sigma$ and $\tau$. An argument similar as above, again referring to 7-largeness of $X$, shows that $s$ and $t$ are adjacent in $X$. The lemma follows then by flagness of $X$.

Proposition 4.7 (see Lemma 3.4 in [8]). In a 7-systolic complex all bigons are 1-thin.

Proof. Let $\left(\gamma, \gamma^{\prime}\right)$ be a bigon in $X$. Let $v$ denote the common origin of $\gamma, \gamma^{\prime}$, and let $w, w^{\prime}$ denote their endpoints. We prove that $\left(\gamma, \gamma^{\prime}\right)$ is 1-thin by induction on $n=d(v, w)=d\left(v, w^{\prime}\right)$.

There is nothing to prove if $n \leq 1$, so let us assume that $n \geq 2$. Let then $s, s^{\prime}$ denote the vertices on $\gamma, \gamma^{\prime}$ just before $w, w^{\prime}$. By induction it suffices to prove that $d\left(s, s^{\prime}\right) \leq 1$.

By Lemma 4.4 there is a geodesic $\gamma^{\prime \prime}$ from $v$ to $w^{\prime}$ such that $\left(\gamma, \gamma^{\prime \prime}\right)$ is a ladder. Let $t, p$ denote the vertices of $\gamma^{\prime \prime}$ at distance $n-2$ and $n-1$ from $v$. By Corollary 4.5 there is a geodesic $\gamma_{1}^{\prime}$ from $v$ to $s^{\prime}$ such that $\left(\gamma^{\prime \prime}, \gamma_{1}^{\prime} .\left(s^{\prime}, w^{\prime}\right)\right)$ is a ladder. Let $t^{\prime}$ denote the vertex of $\gamma_{1}^{\prime}$ just before $s^{\prime}$. Denote by $\sigma$ the simplex spanned by $\left\{w, w^{\prime}\right\}$ (of dimension 1 or 0 ), and by $\tau$ the simplex spanned by $\left\{t, t^{\prime}\right\}$ ( $\tau$ is well-defined because $\left(\gamma^{\prime \prime}, \gamma_{1}^{\prime} .\left(s^{\prime}, w^{\prime}\right)\right)$ is a ladder). Since $t, t^{\prime} \in S_{n-2}\left(v_{0}\right)$ and $w, w^{\prime} \in S_{n}\left(v_{0}\right)$, we have $\tau \cap B(\sigma, X)=\emptyset$.

By Lemma 4.6 the intersection $B(\sigma, X) \cap B(\tau, X)$ is a simplex. Since this intersection contains $s$ and $s^{\prime}$, it follows that $d\left(s, s^{\prime}\right) \leq 1$. This completes the proof.

4.2. Geodesic convexity. In this section we relate the (global) notion of geodesic convexity (see Introduction) to the (local) notion of convexity (see Definition 2.5). 
Lemma 4.8. Let $X$ be a systolic complex, and let $Y \subset X$ denote any convex subcomplex. Assume that $\left(\gamma=\left(v_{0}=v, \ldots, v_{n}=w\right), \gamma^{\prime}=\left(v_{0}^{\prime}=v, \ldots, v_{n}^{\prime}=w\right)\right)$ is a bigon of $X$ such that all vertices of $\gamma$ are in $Y$. Then all vertices of $\gamma^{\prime}$ are in $Y$.

Proof. We argue by induction on the length $n$.

Let $\left(\gamma=\left(v_{0}=v, \ldots, v_{n}, v_{n+1}=w\right), \gamma^{\prime}=\left(v_{0}^{\prime}=v, \ldots, v_{n}^{\prime}, v_{n+1}^{\prime}=w\right)\right)$ denote a bigon of $X$ such that all vertices of $\gamma$ are in $Y$. By Corollary 4.5 there is a geodesic $\gamma_{1}=\left(u_{0}=v_{0}, u_{1}, \ldots, u_{n}=v_{n}^{\prime}\right)$ from $v_{0}$ to $v_{n}^{\prime}$ such that $\left(\gamma, \gamma_{1} \cdot\left(v_{n}^{\prime}, v_{n+1}\right)\right)$ is a ladder. We claim that all vertices of $\gamma_{1}$ are in $Y$. To see this, we look first at $u_{1}$. If $u_{1}=v_{1}$ then $u_{1} \in Y$. Otherwise $\left(v_{0}, u_{1}, v_{2}\right)$ is a path of $X_{v_{1}}$ with endpoints in $Y_{v_{1}}$. Since $v_{0}, v_{2}$ are at distance 2 in $X$, and hence also in $X_{v_{1}}$, by 3 -convexity of $Y_{v_{1}}$ in $X_{v_{1}}$ we have $u_{1} \in Y$. Furthermore, we get two length $n$ geodesics $\left(u_{1}, v_{2}, \ldots, v_{n}, v_{n+1}\right)$ and $\left(u_{1}, \ldots, u_{n}, v_{n+1}\right)$, such that all vertices of the first geodesic belong to $Y$. By induction, all vertices $u_{1}, \ldots, u_{n}$ belong to $Y$ and thus all vertices of $\gamma_{1}$ are in $Y$.

Now, we get another pair of length $n$ geodesics $\gamma_{1}$ and $\left(v_{0}^{\prime}, \ldots, v_{n}^{\prime}\right)$, with common endpoints, such that all vertices of the first geodesic belong to $Y$. By induction, all vertices $v_{1}^{\prime}, \ldots, v_{n}^{\prime}$ belong to $Y$, which finishes the proof.

Proposition 4.9. Let $X$ be a systolic complex, and let $Y \subset X$ be a (non empty) subcomplex. Then $Y$ is convex iff it is geodesically convex.

Proof. First assume that $Y$ is a geodesically convex subcomplex. Then $Y$ is clearly connected. Let us check now that $Y$ is locally 3-convex. Let $v$ be any vertex of $Y$, and let $\left(v_{1}, v_{2}, v_{3}\right)$ denote any path of the link $X_{v}$, such that $v_{1}, v_{3} \in Y_{v}$ and $v_{1}, v_{3}$ are not connected by an edge in $X_{v}$. Then $v_{1}, v_{3}$ are distinct vertices of $X$, and their combinatorial distance cannot be 1 (for otherwise there would be an edge in $X$ joining $v_{1}, v_{3}$ and, by flagness of $X$, this edge would belong to $\left.X_{v}\right)$. Thus $\left(v_{1}, v_{2}, v_{3}\right)$ is a geodesic of $X$. By geodesic convexity we then have $v_{2} \in Y$, and since $Y$ is full by assumption, we also get $v_{2} \in Y_{v}$. Consequently, $Y$ is convex.

To prove the converse, assume that $Y$ is a convex subcomplex of $X$, i.e. it is connected and locally 3-convex. By Lemma 2.6, $Y$ is then full. By Lemma 4.8 we need to prove that for any two vertices $p, q$ in $Y$ there exists a geodesic of $X$, from $p$ to $q$, all of whose vertices belong to $Y$.

Let $p$ be a vertex in $Y$. Put $Y_{0}=\{p\}$, and for each integer $k \geq 1$ we define inductively $Y_{k}$ as the union of all simplices of $Y$ that intersect $Y_{k-1}$. Since $Y$ is connected, it is the union of the subcomplexes $Y_{k}$. Thus it is enough to prove that for each integer $k \geq 0$ and each vertex $q \in Y_{k}$, there exists a geodesic of $X$, from $p$ to $q$, all of whose vertices are contained in $Y$. This fact is straightforward for $k=0$ or $k=1$, and we argue by induction on $k \geq 1$.

Let $q$ be a vertex of $Y_{k+1}$. There exists a vertex $q^{\prime} \in Y_{k}$ such that $\left\{q, q^{\prime}\right\}$ is a simplex of $Y$. In particular $\left|d(p, q)-d\left(p, q^{\prime}\right)\right| \leq 1$, where $d$ denotes the combinatorial 
distance in $X$. By induction, there is a geodesic $\gamma^{\prime}$ from $p$ to $q^{\prime}$ all of whose vertices are contained in $Y$.

If $d(p, q)>d\left(p, q^{\prime}\right)$, then the concatenation $\gamma^{\prime} .\left(q^{\prime}, q\right)$ is a geodesic from $p$ to $q$ all of whose vertices are contained in $Y$. If $d\left(p, q^{\prime}\right)>d(p, q)$, then $q$ is on some geodesic $\gamma$ from $p$ to $q^{\prime}$. Since $\gamma$ and $\gamma^{\prime}$ have the same endpoints, by Lemma 4.8 all the vertices of $\gamma$ are in $Y$. In particular, the initial part of $\gamma$ between $p$ and $q$ is a geodesic with all vertices in $Y$.

Assume now that $d(p, q)=d\left(p, q^{\prime}\right)$. By Lemma 4.4, there exists a geodesic $\gamma$ from $p$ to $q$ such that $\left(\gamma^{\prime}, \gamma\right)$ is a ladder. Let $q^{\prime \prime}$ denote the vertex of $\gamma$ just before $q$, and let $\gamma^{\prime \prime}$ be the initial part of $\gamma$ between $p$ and $q^{\prime \prime}$. Then $q^{\prime \prime}$ is linked to $q^{\prime}$ and $\gamma^{\prime \prime} .\left(q^{\prime \prime}, q^{\prime}\right)$ is a geodesic from $p$ to $q^{\prime}$. It follows by Lemma 4.8 that all the vertices of $\gamma^{\prime \prime}$, and thus also of $\gamma$, are in $Y$, which completes the proof.

Corollary 4.10. The balls of a systolic complex are geodesically convex.

Proof. By Lemma 2.12 the balls are salient and hence convex. Thus, by Proposition 4.9, they are geodesically convex.

\section{Quasi-convexity in 7-systolic complexes}

In this section we show that each quasi-convex subcomplex in a 7-systolic complex is at finite Hausdorff distance of a convex subcomplex (Theorem 5.5).

Lemma 5.1 (Y-lemma). Let $v$ denote a vertex in a systolic complex $X$. Let $v_{1}, v_{2}$ denote vertices at distance $n$ from $v$ and with $d\left(v_{1}, v_{2}\right)=d \leq n$. Then there is a geodesic of length $n-d$ with origin $v$ that extends to a geodesic to either of the vertices $v_{i}$.

Proof. We argue by induction on $d$. The result is obvious for $d=0$.

Let $\left(x_{0}=v_{1}, x_{1}, \ldots, x_{d}=v_{2}\right)$ denote a geodesic. By Corollary 4.10 this geodesic is contained in $B_{n}(v, X)$. If $d\left(v, x_{1}\right)=n$ we choose a vertex $w_{1}$ such that $d\left(v, w_{1}\right)=n-1$ and $w_{1}, x_{0}, x_{1}$ span a simplex. If $d\left(v, x_{1}\right)=n-1$ we set $w_{1}=x_{1}$. We define similarly $w_{2}$ (where now $v_{2}$ plays the role of $v_{1}$ ).

We claim that $d\left(w_{1}, w_{2}\right) \leq d-1$.

To prove the claim we consider two cases. In the first case we assume that the geodesic $\left(x_{0}, x_{1}, \ldots, x_{d}\right)$ does not enter the ball $B_{n-1}(v, X)$. In this case there is a sequence $\left(y_{1}=w_{1}, y_{2}, \ldots, y_{d}=w_{2}\right)$ of vertices of the sphere $S_{n-1}(v, X)$ such that $y_{k}, x_{k-1}, x_{k}$ span a simplex. Then the vertices $y_{k}, y_{k+1}$ span a simplex, since they both belong to the projection of $x_{k}$ on the sphere $S_{n-1}(v, X)$. Consequently, we have $d\left(w_{1}, w_{2}\right) \leq d-1$. 
In the second case we assume that the geodesic $\left(x_{0}, x_{1}, \ldots, x_{d}\right)$ enters the ball $B_{n-1}(v, X)$, so there are integers $1 \leq i \leq j \leq d-1$ such that

$$
d\left(v, x_{0}\right)=d\left(v, x_{1}\right)=\cdots=d\left(v, x_{i-1}\right)=n=d\left(v, x_{j+1}\right)=\cdots=d\left(v, x_{d}\right)
$$

and

$$
d\left(v, x_{i}\right)=d\left(v, x_{j}\right)=n-1 .
$$

Observe that there is a sequence $\left(y_{1}=w_{1}, y_{2}, \ldots, y_{i}=x_{i}\right)$ of vertices of the sphere $S_{n-1}(v, X)$ such that $y_{k}, x_{k-1}, x_{k}$ span a simplex. Similarly, there is a sequence $\left(y_{j}=x_{j}, y_{j+1}, \ldots, y_{d-1}=w_{2}\right)$ of vertices of the sphere $S_{n-1}(v, X)$ such that $y_{k}, x_{k+1}, x_{k}$ span a simplex. As in the previous case, any two subsequent vertices $y_{K}, y_{k+1}$ in any of the sequences $\left(y_{1}, y_{2}, \ldots, y_{i}\right)$ and $\left(y_{j}, y_{j+1}, \ldots, y_{d-1}\right)$ span a simplex, hence we have $d\left(w_{1}, w_{2}\right) \leq(i-1)+(j-i)+(d-j-1)=d-2$. Thus the claim follows.

By induction there is a geodesic of length $(n-1)-d\left(w_{1}, w_{2}\right) \geq n-d$ with origin $v$ that extends to a geodesic to either of the vertices $w_{i}$. Both of those geodesics can be extended to vertices $v_{i}$, which finishes the proof.

The next two results require the assumption of 7-systolicity.

Lemma 5.2. Let $X$ denote a 7 -systolic complex. Let $\left(v_{0}, v_{1}, \cdots, v_{n}\right),\left(v_{0}, v_{1}^{\prime}, \cdots, v_{n}^{\prime}\right)$ denote two geodesics with the same origin and such that $d\left(v_{1}, v_{1}^{\prime}\right)=2$. Then $n<$ $2+d\left(v_{n}, v_{n}^{\prime}\right)$.

Proof. We argue by contradiction. Suppose that $d\left(v_{n}, v_{n}^{\prime}\right) \leq n-2$ (in particular $n \geq 2)$. By Lemma 5.1, there is a geodesic $\left(v_{0}, x, y\right)$ which extends to geodesics from $v_{0}$ to either of the vertices $v_{n}$ and $v_{n}^{\prime}$. Since in 7-systolic complexes geodesic bigons are 1-thin (see Proposition 4.7), we have $d\left(x, v_{1}\right) \leq 1, d\left(x, v_{1}^{\prime}\right) \leq 1, d\left(y, v_{2}\right) \leq 1$ and $d\left(y, v_{2}^{\prime}\right) \leq 1$.

By Proposition 2.2 the complex $X$ is 7-large: we now use this property to find a contradiction. First, note that there is no edge between $v_{1}^{\prime}$ and $v_{2}$. Indeed, if there is such an edge then $\left(v_{0}, v_{1}, v_{2}, v_{1}^{\prime}, v_{0}\right)$ is a cycle of length 4 in $X$ without diagonals. Similarly, there is no edge between $v_{1}$ and $v_{2}^{\prime}$. This also means that $v_{2} \neq v_{2}^{\prime}$. Now, note that there is no edge between $v_{2}$ and $v_{2}^{\prime}$. Indeed, if there is such an edge then $\left(v_{0}, v_{1}, v_{2}, v_{2}^{\prime}, v_{1}^{\prime}, v_{0}\right)$ is a cycle of length 5 in $X$ without diagonals. In particular, we have $y \neq v_{2}$ and $y \neq v_{2}^{\prime}$. But then $\left(v_{0}, v_{1}, v_{2}, y, v_{2}^{\prime}, v_{1}^{\prime}, v_{0}\right)$ is a cycle of length 6 in $X$ without diagonals, contradiction.

Corollary 5.3. Let $v_{1}, v_{2}, x$ be vertices of a 7-systolic complex $X$, and suppose that $d\left(v_{1}, v_{2}\right) \leq d$ and $d\left(v_{1}, x\right)=d\left(v_{2}, x\right)=n \geq d+2$. Denote by $\sigma_{1}, \sigma_{2}$ the projections of the vertex $x$ on the spheres $S_{n-1}\left(v_{1}, X\right)$ and $S_{n-1}\left(v_{2}, X\right)$ respectively. Then $\sigma_{1} \cup \sigma_{2}$ spans a simplex of $X$. 
Proof. Since $X$ is flag, it is enough to check that, given any vertices $w_{1} \in \sigma_{1}$ and $w_{2} \in \sigma_{2}$, these vertices either coincide or are connected with an edge of $X$. We argue by contradiction. Consider vertices $w_{1} \in \sigma_{1}, w_{2} \in \sigma_{2}$ such that $d\left(w_{1}, w_{2}\right)=2$. Then Lemma 5.2 applies to geodesics of length $n$ from $v$ to $v_{1}, v_{2}$ passing through $w_{1}, w_{2}$ respectively. We deduce that $d\left(v_{1}, v_{2}\right)>n-2 \geq d$, which contradicts the assumption.

Definition 5.4. Given $K>0$, we say that a subcomplex $Y$ in a connected simplicial complex $X$ is $K$-quasi-convex whenever the following holds: for any geodesic $\left(x_{0}, \ldots, x_{n}\right)$ in $X$ such that $x_{0} \in Y, x_{n} \in Y$ we have $d\left(x_{i}, Y\right) \leq K$ for $0 \leq i \leq n$. A subcomplex $Y$ is quasi-convex in $X$ if it is $K$-quasi-convex for some $K$.

Theorem 5.5. Let $X$ denote a locally finite dimensional 7-systolic complex. Let $Y \subset X$ be a $K$-quasi-convex subcomplex, for some $K>0$. Then there exists an integer $n(K)$ such that for every $n \geq n(K)$ the neighbourhood $B_{n}(Y, X)$ is salient.

Proof. Since $X$ is 7-systolic, it is Gromov-hyperbolic (see Theorem 2.3 (2)). Thus $X$ admits quasi-projections on quasi-convex subcomplexes (see e.g. Proposition 3.11 on p. 463 in [2]). In particular, there exists an integer $d=d(K) \geq 0$ such that for any vertex $x$ in $X$ and any vertices $v_{1}, v_{2}$ in $Y$, if $d\left(x, v_{1}\right)=d(x, Y)=d\left(x, v_{2}\right)$ then we have $d\left(v_{1}, v_{2}\right) \leq d$.

Now, we set $n(K)=\max (K, d(K)+2)$. Since $n(K) \geq K$ and $Y$ is $K$-quasiconvex, it follows that for any $n \geq n(K)$ the neighbourhood $B_{n}(Y, X)$ is connected. To prove that $B_{n}(Y, X)$ is salient, it remains to check its links at vertices (see Lemma 2.10).

Let $x$ be a vertex of $B_{n}(Y, X)$. If $d(x, Y)<n$, we have $\left[B_{n}(Y, X)\right]_{x}=X_{x}$. Thus, it remains to consider the case when $d(x, Y)=n$. In this case, denote by $\operatorname{Proj}_{Y}(x)$ the set of vertices of $Y$ at distance $n$ from $x$. By the properties of quasi-projections, the set $\operatorname{Proj}_{Y}(x)$ has diameter at most $d(K)$. For each $y \in \operatorname{Proj}_{Y}(x)$, denote by $\sigma_{y}$ the projection of $x$ on the sphere $S_{n-1}(y, X)$. We claim that the set of simplices $\left\{\sigma_{y}: y \in \operatorname{Proj}_{Y}(x)\right\}$ spans a simplex of $X$. Indeed, since $n \geq n(K) \geq d(K)+2$, it follows from Corollary 5.3 that any pair of simplices from this set spans a simplex. The claim then follows by flagness of $X$, and by the fact that $X$ is locally finite dimensional (the latter implies that the set $\left\{\sigma_{y}: y \in \operatorname{Proj}_{Y}(x)\right\}$ is finite, even if $\operatorname{Proj}_{Y}(x)$ is infinite). We denote by $\tau$ the simplex of $X$ spanned by the set $\left\{\sigma_{y}: y \in \operatorname{Proj}_{Y}(x)\right\}$.

Observe that, again due to flagness of $X$, the simplex $\tau$ belongs to the link $X_{x}$. Moreover, using Lemma 2.11 and Lemma 2.15 we get

$$
\left[B_{n}(Y, X)\right]_{x}=\bigcup_{y \in \operatorname{Proj}_{Y}(x)}\left[B_{n}(y, X)\right]_{x}=\bigcup_{y \in \operatorname{Proj}_{Y}(x)} B\left(\sigma_{y}, X_{x}\right)=B\left(\tau, X_{x}\right) .
$$

Thus $B_{n}(Y, X)$ is salient. 
Definition 5.6. Let $\Gamma$ be a word-hyperbolic group. A subgroup $H<\Gamma$ is quasiconvex if $H$ is a quasiconvex subset in the Cayley graph $C(\Gamma, S)$ for some finite generating set $S$.

Recall that, since $\Gamma$ is word-hyperbolic, quasi-convexity does not depend on the choice of the generating set $S$ ([2], Corollary 3.6, p. 461).

Remark 5.7. When $H$ is a quasi-convex subgroup of a word-hyperbolic group $\Gamma$, and $\Gamma$ acts discretely cocompactly on a simplicial complex $X$, then for any compact subcomplex $D \subset X$ the union $Y=\bigcup_{h \in H} h D$ is a quasiconvex subcomplex of $X$.

Indeed, for any vertex $v_{0} \in D$, the map $\gamma \mapsto \gamma v_{0}$ is a quasi-isometry of $\Gamma$ onto $X$ (admitting a quasi-inverse). It follows that the orbit $H\left\{v_{0}\right\}$ is quasiconvex, because quasi-geodesics of $\Gamma$ are at finite Hausdorff distance of a geodesic ([2], Theorem 1.7, p. 401). Now the quasi-convexity of $Y$ follows, since by compactness of $D$ and hyperbolicity of $X$ any geodesic with endpoints in $Y$ is at finite Hausdorff distance of a geodesic with endpoints in some orbit $H\left\{v_{0}\right\}\left(v_{0}\right.$ a vertex of $\left.D\right)$.

Observe that in the next corollary $\Gamma$ is a 7 -systolic group, and hence it is wordhyperbolic (see Corollary 2.4).

Corollary 5.8. Let $X$ be a locally compact 7-systolic complex and let $\Gamma$ be a group acting discretely and cocompactly on $X$. Then any quasiconvex subgroup $H$ is convex cocompact in the following sense: there exists a convex (even salient) subcomplex $Y$ such that $H$ preserves $Y$ and $H$ acts on $Y$ discretely and cocompactly.

Proof. Since $H$ is quasiconvex for any fixed vertex $v_{0}$ the subcomplex $Z=H .\left\{v_{0}\right\}$ is quasiconvex. Thus by Theorem 5.5 for some large $n$ the subcomplex $Y=B_{n}(Z, X)$ is salient. Since $Z$ is $H$-invariant, so is $Y$. And since $H$ is cocompact on $Y$ and balls in $X$ are compact, $H$ is also cocompact on $Y$.

\section{QCS for 7-systolic simplices of groups}

In this section we prove the main result of the paper, Theorem 6.1. We also give some explicit examples of groups to which this theorem applies.

Theorem 6.1. Let $\mathscr{E}$ be a locally 7-large, locally extra-tilable simplex of finite groups. Then any quasi-convex subgroup of the fundamental group of $\mathcal{E}$ is separable.

Proof. Let $X$ denote the universal cover and $\Gamma$ the fundamental group of $\mathscr{E}$. By Proposition 3.6 the simplicial complex $X$ is then 7-systolic and $\Gamma$ acts on it properly discontinuously, cocompactly, and in a locally extra-tilable way. 
Let $H$ be any quasi-convex subgroup of $\Gamma$. We want to show that $H$ is an intersection of finite index subgroups. That is, we need to show that for any element $g \in \Gamma \backslash H$ there is a finite index subgroup $\Gamma^{\prime}<\Gamma$ such that $H<\Gamma^{\prime}$ and $g \notin \Gamma^{\prime}$.

Let $\Delta$ be a top-dimensional simplex of $X$. Consider the subcomplex $Z$ of $X$ being the union of all simplices in the orbit of $\Delta$ under $H . Z$ is then a quasi-convex subcomplex of $X$ by Remark 5.7. Let $g$ be any element of $\Gamma \backslash H$. There exists an integer $n$ such that the subcomplex $Y=B_{n}(Z, X)$ contains the simplex $g \Delta$. By Theorem 5.5, choosing $n$ larger if necessary, we may assume that $Y$ is salient. It is also clear that $H$ preserves $Y$, and that $H$ is cocompact on $Y$ (by local finiteness of $X$ ). By Theorem 3.4, $Y$ is a strict fundamental domain for the subgroup $\Gamma(Y) \subset \Gamma$ generated by the stabilizers of maximal simplices inside $\partial Y$. Since $H$ preserves $Y$, it acts by conjugation on this family of stabilizers. Thus $H$ acts by conjugation on $\Gamma(Y)$. Let $\Gamma^{\prime} \subset \Gamma$ denote the semi-direct product of $\Gamma(Y)$ and $H$. We claim that $\Gamma^{\prime}$ is a finite index subgroup of $\Gamma$ and that $\Gamma^{\prime}$ does not contain $g$.

First, since $\Gamma$ acts simply-transitively on the set $\mathcal{C}$ of top dimensional simplices in $X$, the index $\left[\Gamma: \Gamma^{\prime}\right]$ is equal to the number of orbits of $\Gamma^{\prime}$ in $\mathcal{C}$. Since $Y$ is a (strict) fundamental domain for $\Gamma(Y)$, every such $\Gamma^{\prime}$-orbit meets the set $\mathcal{C}(Y)$ of top dimensional simplices contained in $Y$. Since $H$ is cocompact on $Y$, it is cofinite on $\mathcal{C}(Y)$, and thus there are finitely many $\Gamma^{\prime}$-orbits in $\mathcal{C}$, so $\left[\Gamma: \Gamma^{\prime}\right]<\infty$.

We turn to proving that $g \notin \Gamma^{\prime}$. If, on the contrary, we have $g \in \Gamma^{\prime}$, then $g=\gamma h$ for some $\gamma \in \Gamma(Y)$ and $h \in H$. We also have $g \Delta=\gamma h \Delta$, and thus both $h \Delta$ and $\gamma(h \Delta)$ are contained in $Y$. Since $Y$ is a strict fundamental domain for $\Gamma(Y)$, and since $\gamma \in \Gamma(Y)$, we have $\gamma(h \Delta)=h \Delta$. This implies that $\gamma=1$, because top-dimensional simplices of $X$ have trivial stabilizers under $\Gamma$. Consequently, $g=h$ and thus $g \in H$, which contradicts the assumption.

Remark 6.2. The proof above shows in fact that every quasi-convex subgroup $H<$ $\Gamma$ is an algebraic retract $\Gamma^{\prime} \rightarrow H$ (with kernel $\Gamma(Y)$ ), where $\Gamma^{\prime}<\Gamma$ is a finite index subgroup. And it is well-known that such a virtual retract is separable (see for instance [10]).

As we have already mentioned, simplices of groups satisfying the assumptions of the above theorem exist in abundance in every dimension (Proposition 3.7). As it is shown in [12] (Corollary 19.3(1)), the fundamental groups of simplices of groups as above may have arbitrarily large (virtual) cohomological dimension.

In the remaining part of this section we will show explicit examples in dimension 2.

Example 6.3 (some triangular Coxeter groups). Given positive integers $m_{1}, m_{2}, m_{3}$ such that $\frac{1}{m_{1}}+\frac{1}{m_{2}}+\frac{1}{m_{3}}<1$ consider some hyperbolic triangle $T\left(m_{1}, m_{2}, m_{3}\right)$ with angles $\frac{\pi}{m_{1}}, \frac{\pi}{m_{2}}, \frac{\pi}{m_{3}}$. Let $W\left(m_{1}, m_{2}, m_{3}\right)$ denote the group of isometries of the 
hyperbolic plane generated by the reflections in the sides of $T\left(m_{1}, m_{2}, m_{3}\right)$, and let

$$
X=\bigcup_{w \in W\left(m_{1}, m_{2}, m_{3}\right)} w T\left(m_{1}, m_{2}, m_{3}\right)
$$

denote the associated tesselation of $\mathbb{H}^{2}$. It is clear that $T\left(m_{1}, m_{2}, m_{3}\right)$ is a strict fundamental domain for the action of $W\left(m_{1}, m_{2}, m_{3}\right)$ on $X$.

The links of vertices in $X$ of corresponding types are $2 m_{i}$-gons, so $X$ is 7-systolic iff each $m_{i}$ is $\geq 4$. Moreover, the stabilizers of vertices are the dihedral groups $D_{2 m_{i}}$ of order $2 m_{i}$, with standard actions on the corresponding links. It is then easy to observe that and the action of $W\left(m_{1}, m_{2}, m_{3}\right)$ is locally extra-tilable iff each $m_{i}$ is divisible by 6. By Theorem 6.1, we deduce that the Coxeter group $W=$ $\left\langle t_{1}, t_{2}, t_{3} \mid t_{1}^{2}, t_{2}^{2}, t_{3}^{2},\left(t_{1} t_{2}\right)^{6 k_{3}},\left(t_{2} t_{3}\right)^{6 k_{1}},\left(t_{1} t_{3}\right)^{6 k_{2}}\right\rangle$ is QCS (for any choice of positive integers $\left.k_{1}, k_{2}, k_{3}\right)$.

Any surface group is commensurable to a group $W$ as in Example 6.3. Since the property QCS is commensurability invariant, we have reproved the following result of Scott [17] (by essentially the same method).

Corollary 6.4. Any surface group is QCS.

Before giving a somewhat thicker two-dimensional example, we describe an extratilable, chamber-transitive action on a graph. We refer to [2] for the vocabulary on simple complexes of groups.

Example 6.5 $\left(\left(Z_{2}, Z_{3}\right)\right.$-group). Let $E$ be the 1 -simplex with vertices $v_{1}, v_{2}$. Denote by $\mathcal{F}$ the simplex of groups over $E$ with vertex groups $G_{v_{1}}=Z_{2}$ and $G_{v_{2}}=Z_{3}$, and with $G_{E}=\{1\}$ (so that $\mathscr{F}$ is $\partial$-supported). We will describe a morphism $m: \mathscr{F} \rightarrow T$ to certain group $T$ of order 216 .

Let $\Pi$ be the euclidean plane tesselated by regular hexagons. Denote by $a, b, c$, $d, e, f$ the vertices (in cyclic order) of a chosen hexagon in $\Pi$. Consider the group $A=Z \oplus Z$ of automorphisms of $\Pi$ generated by translations through vectors $6 \cdot \overrightarrow{a c}$ and $6 \cdot \overrightarrow{a e}$. Then the quotient $\Pi / A$ is a torus tesselated by 36 hexagons.

For every vertex $u$ of $\Pi$ rotations through angles $2 \pi / 3$ and $4 \pi / 3$ around $u$ are clearly automorphisms of $\Pi$. Since the group $A$ is preserved by conjugation through these rotations, they are also automorphisms of the quotient $\Pi / A$. Similarly, for a center $s$ of any edge $\epsilon$ of $\Pi$, reflection with respect to the straight line containing $s$ and orthogonal to $\epsilon$ is an automorphism of $\Pi$ that induces an automorphism of the quotient $\Pi / A$. Let $T$ be the group of automorphisms of $\Pi / A$ generated by rotations and reflections as above.

Let $P$ be the barycentric subdivision of the 1 -skeleton of $\Pi / A$. Then $T$ acts by automorphisms on $P$. It is not hard to realize that in fact the action of $T$ on $P$ is without inversions and simply transitive on the edges of $P$. Consequently, any edge $\varepsilon$ 
of $P$ is a strict fundamental domain for $T$. Moreover, the stabilizer of the vertex of $\varepsilon$, say $u$, which is also a vertex of $\Pi / A$, is isomorphic to $Z_{3}$. The stabilizer of the other vertex, say $s$, is isomorphic to $Z_{2}$.

Now define morphism $m: \mathcal{F} \rightarrow T$ by mapping $G_{v_{1}}$ on the stabilizer of $s$ and $G_{v_{2}}$ on the stabilizer of $u$. Then the development $D(\mathcal{F}, m)$ corresponding to this morphism is equivariantly isomorphic to $P$. It is not hard to see that $P$ is 12-large (in particular, it is also 7-large). We leave it as an exercise to check that $P$ (identified as the development $D(\mathscr{F}, m))$ is extra-tilable. It is the smallest known to us extra-tilable development of $\mathscr{F}$.

We now use the previous graph as one of the local developments for a 2-simplex of groups.

Example 6.6 $\left(\left(Z_{2}, Z_{2}, Z_{3}\right)\right.$-groups $)$. Let $\Delta$ be the 2 -simplex with vertices $v_{0}, v_{1}, v_{2}$. Given an integer $k \geq 1$, consider the simplex of groups $\mathscr{E}$ over $\Delta$ with local groups $G_{\Delta}=\{1\}, G_{\left(v_{0}, v_{i}\right)}=Z_{2}$ for $i=1,2, G_{\left(v_{1}, v_{2}\right)}=Z_{3}, G_{v_{0}}=D_{6 k}, G_{v_{i}}=T$ for $i=1,2$ (where $T$ is the group from Example 6.5), and with structure homomorphisms as in $m$ (see Example 6.5) at vertices $v_{1}, v_{2}$, and such that $\left\langle G_{\left(v_{0}, v_{1}\right)}, G_{\left(v_{0}, v_{2}\right)}\right\rangle=G_{v_{0}}$.

The local developments of $\mathcal{E}$ at vertices are then isomorphic to a $2 k$-gon (at $v_{0}$ ) and to $D(\mathcal{F}, m)=P$ (at $v_{1}$ and $v_{2}$ ). In particular, $\mathcal{E}$ is locally 7-large and locally extra-tilable. Consequently, in view of Theorem 6.1, the fundamental group $\pi_{1} \mathscr{E}$ is QCS.

\section{References}

[1] I. Agol, D. D. Long, and A. W. Reid, The Bianchi groups are separable on geometrically finite subgroups. Ann. of Math. (2) 153 (2001), 599-621. Zbl 1067.20067 MR 1836283

[2] M. R. Bridson and A. Haefliger, Metric spaces of non-positive curvature. Grundlehren Math. Wiss. 319, Springer-Verlag, Berlin 1999. Zbl 0988.53001 MR 1744486

[3] M. Burger and S. Mozes, Finitely presented simple groups and products of trees. C. R. Acad. Sci. Paris Sér. I Math. 324 (1997), 747-752. Zbl 0966.20013 MR 1446574

[4] A. Blass and P. M. Neumann, An application of universal algebra in group theory. Michigan Math. J. 21 (1974), 167-169. Zbl 0292.20033 MR 0364475

[5] R. Gitik, Graphs and separability properties of groups. J. Algebra 188 (1997), 125-143. Zbl 0874.20013 MR 1432351

[6] R. Gitik, Doubles of groups and hyperbolic LERF 3-manifolds. Ann. of Math. (2) 150 (1999), 775-806. Zbl 0944.20023 MR 1740992

[7] M. Hall, Jr., Coset representations in free groups. Trans. Amer. Math. Soc. 67 (1949), 421-432. Zbl 0035.01301 MR 0032642

[8] F. Haglund, Complexes simpliciaux hyperboliques de grande dimension. Orsay preprint 2003-71, 2003. http://www.math.u-psud.fr/ biblio/ppo/2003/fic/ppo_2003_71.pdf 
[9] F. Haglund, Finite index subgroups of graph products. Geom. Dedicata, to appear.

[10] F. Haglund and D. T. Wise, Special cube complexes. Geom. Funct. Anal. 17 (2008), 1551-1620.

[11] T. Hsu and D. T. Wise, Separating quasiconvex subgroups of right-angled Artin groups. Math. Z. 240 (2002), 521-548. Zbl 1006.20028 MR 1924020

[12] T. Januszkiewicz and J. Świątkowski, Simplicial nonpositive curvature. Publ. Math. Inst. Hautes Études Sci. 104 (2006), 1-85. Zbl 05117094 MR 2264834

[13] I. Kapovich and D. T. Wise, The equivalence of some residual properties of wordhyperbolic groups. J. Algebra 223 (2000), 562-583. Zbl 0951.20029 MR 1735163

[14] W. Magnus, Residually finite groups. Bull. Amer. Math. Soc. 75 (1969), 305-316. Zbl 0196.04704 MR 0241525

[15] A. I. Mal'cev, On homomorphisms onto finite groups. Uch. Zap. Ivanov. Gos. Pedagog Inst. 18 (1958), 49-60; English transl. Amer. Math. Soc. Transl. (2) 119 (1983), 67-79. Zbl 0511.20026

[16] V. Metaftsis and E. Raptis, Subgroup separability of graphs of nilpotent groups. J. Group Theory 7 (2004), 265-273. Zbl 1063.20026 MR 2049021

[17] P. Scott, Subgroups of surface groups are almost geometric. J. London Math. Soc. (2) 17 (1978), 555-565; Correction to 'Subgroups of surface groups are almost geometric', ibid. 32 (1985), 217-220. Zbl 0412.57006 MR 0494062; Zbl 0581.57005 MR 0811778

[18] D. T. Wise, Non-positively curved squared complexes, aperiodic tilings, and nonresidually finite groups. PhD Thesis, Princeton University, Princeton 1996.

[19] D. T. Wise, Subgroup separability of graphs of free groups with cyclic edge groups. Q.J. Math. 51 (2000), 107-129. Zbl 0991.05056 MR 1760573

[20] D. T. Wise, The residual finiteness of negatively curved polygons of finite groups. Invent. Math. 149 (2002), 579-617. Zbl 1040.20024 MR 1923477

[21] D. T. Wise, Sixtolic complexes and their fundamental groups. In preparation.

Received June 26, 2006

F. Haglund, Laboratoire de Mathématiques, Université de Paris XI (Paris-Sud), 91405 Orsay, France

E-mail: frederic.haglund@math.u-psud.fr

J. Świątkowski, Instytut Matematyczny, Uniwersytet Wrocławski, pl. Grunwaldzki 2/4, 50-384 Wrocław, Poland

E-mail: swiatkow@math.uni.wroc.pl 